\title{
The Difference in Pharmacokinetics and Pharmacodynamics between Extended-Release Fluvastatin and Immediate-Release Fluvastatin in Healthy Chinese Subjects
}

\author{
H. R. Xu, W. L. Chen, N. N. Chu, X. N. Li, and J. R. Zhu \\ Department of Clinical Pharmacology, Zhongshan Hospital, Fudan University, Shanghai 200032, China \\ Correspondence should be addressed to X. N. Li, li.xuening@zs-hosputal.sh.cn
}

Received 7 February 2012; Revised 28 April 2012; Accepted 14 May 2012

Academic Editor: Kazim Husain

Copyright $\odot 2012$ H. R. Xu et al. This is an open access article distributed under the Creative Commons Attribution License, which permits unrestricted use, distribution, and reproduction in any medium, provided the original work is properly cited.

\begin{abstract}
The aim of this study was to evaluate the difference in pharmacokinetics and pharmacodynamics between extended-release (ER) fluvastatin tablet and its immediate-release (IR) capsule in Chinese healthy subjects. This was an open-label, single/multiple-dose, two-period, two-treatment, crossover, randomized trial with a minimum washout period of 7 days. Twenty healthy male adult subjects were given fluvastatin ER tablet $80 \mathrm{mg}$ QD by oral administration or fluvastatin IR capsule $40 \mathrm{mg}$ BID for seven days. Blood samples were collected up to 24 hours after dosing on day 1 and day 7. Serum concentrations of fluvastatin were determined by LC-MS/MS. For fluvastatin ER tablet $80 \mathrm{mg} \mathrm{QD}, C_{\max }$ was $61.0 \pm 39.0$ and $63.9 \pm 29.7 \mathrm{ng} / \mathrm{mL}$, and $\mathrm{AUC} \mathrm{C}_{0-24 \mathrm{~h}} \mathrm{was}_{242} \pm 156$ and $253 \pm 91.1 \mathrm{ng} \cdot \mathrm{h} / \mathrm{mL}$ on day 1 and 7, respectively. For fluvastatin IR capsule $40 \mathrm{mg}$ BID, $C_{\max }$ was $283 \pm 271 \mathrm{and} 382 \pm 255 \mathrm{ng} / \mathrm{mL}$, and $\mathrm{AUC}_{0-24 \mathrm{~h}}$ was $720 \pm 776$ and $917 \pm 994 \mathrm{ng} \cdot \mathrm{h} / \mathrm{mL}$ on day 1 and day 7 , respectively. The relative bioavailability of fluvastatin ER tablet $80 \mathrm{mg}$ QD to fluvastatin IR capsule $40 \mathrm{mg}$ BID is $(45.3 \pm 23.9) \%$ and $(43.3 \pm 24.1) \%$ on day 1 and day 7 , respectively. $T_{\max }$ for fluvastatin ER tablet was 2.50 and $2.60 \mathrm{~h}$ and for capsule was 0.78 and $0.88 \mathrm{~h}$ on day 1 and day 7 , respectively. In the first period, compared to baseline, cholesterol decreased $15.3 \%$ in fluvastatin ER tablet $80 \mathrm{mg}$ QD and $16.9 \%$ in fluvastatin IR capsule $40 \mathrm{mg}$ BID. Triglyceride decreased 3.7\% in fluvastatin ER tablet $80 \mathrm{mg}$ QD and 19.1\% in fluvastatin IR capsule $40 \mathrm{mg}$ BID. The difference has no statistical significance at $P>0.05$ in reduction percent of cholesterol and triglyceride between the two groups. No adverse events were recorded. The results indicated that $C_{\max }$ of fluvastatin ER tablet is reduced and $T_{\max }$ is prolonged compared with IR capsule. There is no accumulation for ER formulation after multiple doses.
\end{abstract}

\section{Introduction}

Fluvastatin, the 3-hydroxy-3-methylglutaryl coenzyme A reductase inhibitor, is the first fully synthetic HMG-CoA reductase inhibitor and the well-established first-line agent for the treatment of hypercholesterolemia, primarily due to its marked lowering action of low-density lipoprotein cholesterol (LDL-C) levels (as discussed by Bruckert et al. [1]). The benefits of statin therapy on cardiovascular morbidity and mortality are thought to be related to both LDL-C lowering and pleiotropic effects such as modulation of inflammatory protein levels (as discussed elsewhere [2, 3]). Small increases in HDL-C and variable decrease in TG are also observed.
Fluvastatin is relatively hydrophilic, compared with the semisynthetic HMG-CoA reductase inhibitors, and, therefore, it is extensively absorbed from the gastrointestinal tract. After absorption, it is nearly completely extracted and metabolized in the liver to 2 hydroxylated metabolites and an $\mathrm{N}$-desisopropyl metabolite, which are excreted in the bile. Approximately $95 \%$ of a dose is recovered in the faeces, with $60 \%$ of a dose recovered as the 3 metabolites (as discussed by Scripture and pieper [4]). An ER formulation of fluvastatin was developed as a starting and maintenance treatment for once-daily administration. It uses a hydrophilic cellulose matrix that swells when it is in contact with fluid in the intestine. Fluvastatin then diffuses through the matrix and is released over an 8 -hour period. It was hypothesized that 


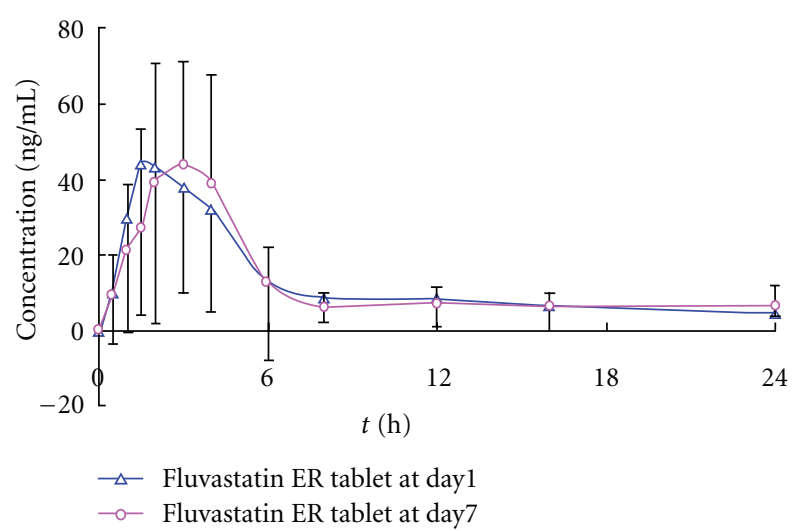

Figure 1: The mean serum concentration-time curves after single and multiple doses of fluvastatin extended-release tablet in Chinese healthy volunteers $(n=20)$.

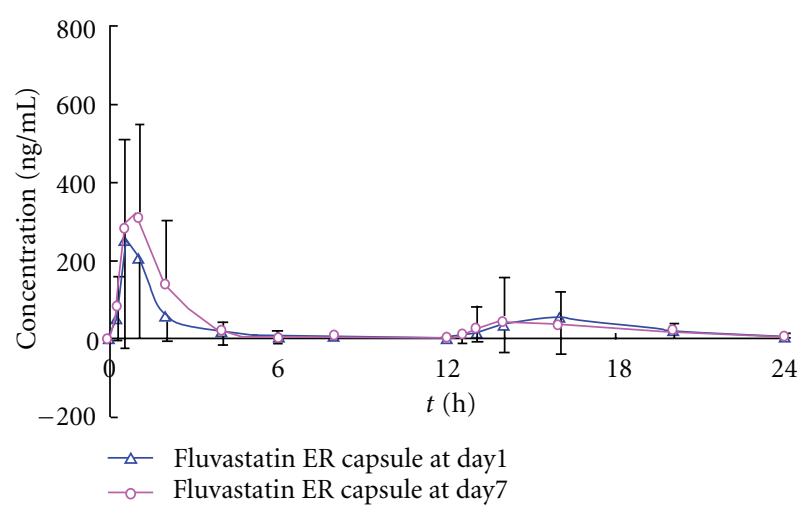

FIgURE 2: The mean serum concentration-time curves after single and multiple doses of fluvastatin immediate-release capsule in Chinese healthy volunteers $(n=20)$.

this new formulation would avoid hepatic saturation without elevating systemic drug levels and thus provide greater efficacy compared with the conventional formulation (as discussed elsewhere [5, 6]). Fluvastatin ER tablet $80 \mathrm{mg} / \mathrm{d}$ has been reported to provide comprehensive lipid management with a good safety profile similar to that of placebo in clinical trials (as discussed by Battantyne et al. [7]).

The pharmacokinetic properties of two fluvastatin formulations have been investigated in healthy white adult subjects (as discussed by Isaacsohn et al. [5]). Fluvastatin systemic exposure was less than $50 \%$ when administered as fluvastatin ER $80 \mathrm{mg}$ qd compared with fluvastatin IR $40 \mathrm{mg}$ BID (as discussed by Barilla et al. [6]). There is no pharmacokinetic data for fluvastatin ER tablet in Chinese subjects.

The purpose of the present study was to evaluate the difference in pharmacokinetics and pharmacodynamics between extended-release (ER) fluvastatin tablet and its immediate-release (IR) capsule after single and multiple doses in Chinese healthy subjects living in China. The study was conducted in accordance with the regulatory requirements for registration in China.

\section{Materials and Methods}

2.1. Study Populations. Twenty healthy male adult subjects were invited to participate in an open-label, randomized, single and multiple dose, two-period, two-treatment, crossover study. At the time of enrolment, the subjects were informed of the purpose, duration, and risks of the study, and they were requested to sign a written informed consent. They were also informed about the possibility of withdrawing from the study. They were prohibited to take food that contains xanthine or beverages from $48 \mathrm{~h}$ prior to the study until the end of the study. The subjects were also instructed to abstain from taking any medication except paracetamol in 2 weeks prior to the study. The evaluations and tests performed included physical examination, body height, body weight, vital signs (heart rate, systolic and diastolic blood pressure, and body temperature), hematology, blood chemistry, urinalysis, and electrocardiogram. The subjects were requested to report any abnormality occurring throughout and after the study. The results of clinical evaluations were documented in individual case report forms. The study protocol was approved by the Ethics Committee of Zhongshan Hospital.

2.2. Study Design. Healthy subjects were randomized in two groups of ten subjects, and each group received the two drug treatments at two different periods, with a 7-day interdose washout periods. Each volunteer received fluvastatin ER tablet $80 \mathrm{mg}$ QD or fluvastatin IR capsule $40 \mathrm{mg}$ BID for seven days. Each formulation was administered with $200 \mathrm{~mL}$ of water to subjects after a $10 \mathrm{~h}$ overnight fasting. Lunch and dinner were served at 4 and $10 \mathrm{~h}$ after dose, respectively. If the subject is receiving the fluvastatin IR BID treatment, the evening dose of fluvastatin IR $40 \mathrm{mg}$ will be administered 12 hours following the morning dose. The evening meal should be consumed 3 hours prior to this dose. Blood samples were collected through an intravenous catheter in the Lescol IR $40 \mathrm{mg}$ group before dose and at $0.25,0.5,1,2,4,6,8,12$, $12.5,13,14,16,20$, and $24 \mathrm{~h}$ after dose on day 1 and day 7. And for fluvastatin $80 \mathrm{mg}$ ER tablet group, blood samples were drawn prior to dose and at $0.5,1,1.5,2,3,4,6,8,12,16$, $24 \mathrm{~h}$ following dose on day 1 and day 7 . The blood samples were transferred to tubes and centrifuged $\left(4^{\circ} \mathrm{C}\right)$ at $800 \mathrm{rpm}$ for $15 \mathrm{~min}$. The serum samples were stored at $-20^{\circ} \mathrm{C}$ until chromatographic analysis.

\subsection{Determination of Serum Fluvastatin Concentrations.} Serum fluvastatin concentrations were determined by using an LC-MS/MS method developed by personnel at Zhongshan Hospital, Shanghai, China, in which 6-methylfluvastatin sodium was used as the internal standard. The method included $0.2 \mathrm{~mL}$ serum, $0.1 \mathrm{~mL}$ of internal standard solution(6-methyl-fluvastatin $200 \mathrm{ng} / \mathrm{mL}$ ), $0.4 \mathrm{~mL}$ saturation sodium chloride solution, and $0.8 \mathrm{~mL}$ acetonitrile. These constituents were mixed together in a $2.0 \mathrm{~mL}$ centrifuge tube for $30 \mathrm{~s}$. The tube was centrifuged for $15 \mathrm{~min}$ at $18000 \mathrm{rpm}$ and $4^{\circ} \mathrm{C}$. The supernatant was filtered by $0.45 \mu \mathrm{m}$ membrane and injected into the chromatographic system (SIL-HTc, Shimadzu Corporation, Kyoto, Japan). Fluvastatin concentration was determined with a $5.0 \mathrm{~cm} \times 3.0 \mathrm{~mm}$ 
TABle 1: Pharmacokinetic parameters of fluvastatin of two fluvastatin formulations after single and multiple doses in healthy volunteers $(\mathrm{Mean} \pm \mathrm{SD})$.

\begin{tabular}{|c|c|c|c|}
\hline & Parameter & Test (extended-release tablet) & Reference (immediate-release capsule) \\
\hline \multirow{5}{*}{ Single dose } & $C_{\max }(\mathrm{ng} / \mathrm{mL})$ & $61.0 \pm 39.0$ & $283 \pm 271$ \\
\hline & $T_{\max }(\mathrm{h})$ & $2.50 \pm 1.34$ & $0.78 \pm 0.38$ \\
\hline & $\operatorname{MRT}(\mathrm{h})$ & $4.98 \pm 2.28$ & $1.45 \pm 0.44$ \\
\hline & $\mathrm{AUC}_{0-24}(\mathrm{ng} \cdot \mathrm{h} / \mathrm{mL})$ & $242 \pm 156$ & $720 \pm 776$ \\
\hline & $F(\%)$ & $45.3 \pm 23.9$ & \\
\hline \multirow{9}{*}{ Multiple doses } & $C_{\max }(\mathrm{ng} / \mathrm{mL})$ & $63.9 \pm 29.7$ & $382 \pm 255$ \\
\hline & $C_{\min }(\mathrm{ng} / \mathrm{mL})$ & $1.94 \pm 4.16$ & $0.574 \pm 1.63$ \\
\hline & $C_{\mathrm{av}}(\mathrm{ng} / \mathrm{mL})$ & $12.2 \pm 7.76$ & $52.5 \pm 45.2$ \\
\hline & $T_{\max }(\mathrm{h})$ & $2.60 \pm 0.87$ & $0.88 \pm 0.36$ \\
\hline & MRT (h) & $5.57 \pm 2.40$ & $1.43 \pm 0.30$ \\
\hline & $\mathrm{AUC}_{0-24}(\mathrm{ng} \cdot \mathrm{h} / \mathrm{mL})$ & $253 \pm 91.1$ & $917 \pm 994$ \\
\hline & $R$ & $1.39 \pm 0.77$ & $2.07 \pm 1.42$ \\
\hline & $\mathrm{DF}(\%)$ & $566 \pm 214$ & $811 \pm 213$ \\
\hline & $F(\%)$ & $43.3 \pm 24.1$ & \\
\hline
\end{tabular}

internal diameter column of $3.5 \mu \mathrm{m}$ particle size (XTerra RP18 column, Shiseido Corporation, Tokyo, Japan) and eluted with a mobile phase consisting of a mixture of $1 \%$ formic acid in water and methanol $(25: 75 \mathrm{v} / \mathrm{v})$. The column temperature was $40^{\circ} \mathrm{C}$. Flow rate was maintained at $0.30 \mathrm{~mL} / \mathrm{min}$, and fluvastatin was detected by $\mathrm{MS} / \mathrm{MS}$ detector (Sciex API 3000, Foster City, CA, USA). The mass analyzer was operated in multiple reaction mode; $\mathrm{m} / \mathrm{z}$ for fluvastatin and 6-methyl-fluvastatin were $410.2 \rightarrow 348.1$ and $424.2 \rightarrow 362.0$, respectively. Typical retention time for fluvastatin and internal standard were 2.16 and $2.52 \mathrm{~min}$, respectively. Data acquisition and analysis were carried out using Sciex Analyst 1.4 software. The fluvastatin peak area was used for quantification. Under these conditions, the method was linear in the range of 2 to $2000 \mathrm{ng} / \mathrm{mL}$ (2, 5, $10,20,50,100,200,500,1000,2000 \mathrm{ng} / \mathrm{mL})$. Accuracy was between $91.50 \%$ and $100.85 \%$; the relative SD (RSD) of the method was always $<11 \%$. The method was considered suitable by study investigators for the pharmacokinetic study of fluvastatin.

2.4. Pharmacokinetic and Pharmacodynamic Evaluation and Statistical Analyses. Pharmacokinetic parameters were calculated by a noncompartmental method. The actual time of sample collection was used for pharmacokinetic analyses of fluvastatin ER tablet and IR capsule. The area under the serum concentration versus time curve $\mathrm{AUC}_{0-t}$ was obtained by the linear trapezoidal method. Mean residence time (MRT) is the average total time of a given dose spent in the body, which may be calculated as MRT = AUMC/AUC. The average steady-state concentration $\left(C_{\mathrm{av}}\right)$ is calculated as $C_{a v}$ $=\mathrm{AUC}_{0-\tau} / \tau$. The $C_{\max }$ and $T_{\max }$ were obtained directly from the data. The degree of peak-trough fluctuation at steady state (DF) was calculated using the following equation: $\mathrm{DF}(\%)=\left(C_{\mathrm{max}, \mathrm{ss}}-C_{\min , \mathrm{ss}}\right) / C_{\mathrm{av}, \mathrm{ss}} \times 100 \%$, where $C_{\max , \mathrm{ss}}$, $C_{\mathrm{min}, \mathrm{ss}}$, and $C_{\mathrm{av}, \mathrm{ss}}$ are the maximum, minimum, and average serum concentration, respectively, during a dosing interval at steady state. The accumulation ratio $(R)$ was calculated as $C_{\max , s s} / C_{\max }$. An accumulation ratio of 1 indicated no or minimal accumulation.

Bioavailability is the rate and extent to which the active ingredient or active moiety is absorbed from a drug product and was performed using the $F$ ratio, where $F$ was the ratio of $\mathrm{AUC}_{0-24}$ for fluvastatin ER tablet versus fluvastatin IR capsule. Ratios of $\ln \left(C_{\max }\right)$ and $\ln \left(\mathrm{AUC}_{0-t}\right)$ for all formulations were calculated, and $90 \%$ CIs were obtained. An analysis of variance (ANOVA, SPSS16.0) for cholesterol and triglyceride was carried out to evaluate the pharmacodynamic difference between fluvastatin ER tablet and fluvastatin IR capsule after continuous 7-day administration.

\section{Results and Discussion}

3.1. Pharmacokinetic Results. A total of 20 subjects (male; mean (SD) age, 22.6 (1.1) years (range, 21-25 years); weight, 65.6 (7.6) $\mathrm{kg}(56-80 \mathrm{~kg})$; height, $1.74(0.62) \mathrm{m}(1.62-1.85 \mathrm{~m})$; BMI, $\left.21.7(1.7) \mathrm{kg} / \mathrm{m}^{2}\left(18.7-24.0 \mathrm{~kg} / \mathrm{m}^{2}\right)\right)$ were included in and completed the study. Ten subjects received fluvastatin ER tablet once daily first, and the others received fluvastatin IR capsule twice daily first.

Mean serum concentration-time curves of fluvastatin formulations on day 1 and day 7 are shown in Figures 1 and 2, and the pharmacokinetic properties $\left(C_{\max }, T_{\max }\right.$, MRT, $\left.\mathrm{AUC}_{0-24}\right)$ are summarized in Table 1 . Ninety percent confidence intervals of $\mathrm{AUC}_{0-24}$ ratios were 0.306, 0.494 (ratio 0.388) and 0.293, 0.466 (ratio 0.370) for fluvastatin ER tablet versus fluvastatin IR capsule on day 1 and at day 7 , respectively. Ninety percent confidence intervals of $C_{\max }$ ratios were $0.184,0.376$ (ratio 0.263 ) and $0.158,0.233$ (ratio 0.192) for fluvastatin ER tablet versus fluvastatin IR capsule on day 1 and at day 7, respectively.

3.2. Pharmacodynamic Results. The mean baseline of cholesterol and triglyceride of ten subjects who received fluvastatin 
ER tablet once daily first was $3.70 \pm 0.70$ and $0.67 \pm$ $0.16 \mathrm{mmol} / \mathrm{l}$. The mean reduction of cholesterol and triglyceride after continuous 7 -day administration was $0.58 \pm 0.24$ and $0.05 \pm 0.16 \mathrm{mmol} / \mathrm{l}$, respectively. The percent of reduction was $15.3 \pm 5.4 \%$ and $3.7 \pm 19.8 \%$, respectively. The mean baseline of cholesterol and triglyceride of ten subjects who received fluvastatin IR capsule twice daily first was $3.84 \pm 0.87$ and $0.87 \pm 0.39 \mathrm{mmol} / \mathrm{l}$. The mean reduction of cholesterol and triglyceride after continuous 7 -day administration was $0.71 \pm 0.56$ and $0.23 \pm 0.31 \mathrm{mmol} / \mathrm{l}$, respectively. The percent of reduction was $16.9 \pm 11.2 \%$ and $19.1 \pm 20.4 \%$, respectively. The difference has no statistical significance at $P>0.05$ in baseline of cholesterol and triglyceride between group of $80 \mathrm{mg}$ fluvastatin ER tablet and group of $40 \mathrm{mg}$ fluvastatin IR capsule. The difference has also no statistical significance at $P=0.681$ and $P=0.104$ in reduction percent of cholesterol and triglyceride between the two groups, respectively.

\subsection{Tolerability. No adverse events occurred throughout the} study.

3.4. Discussion. The ER formulation of fluvastatin was developed to decrease the frequency of dose administration and to reduce the incidence of AEs by reducing peak serum concentrations after drug administration. Because these concentrations may be related to the extent in which patients experience common AEs, the use of an ER formulation may help reduce these effects and improve tolerability.

Results from the single and multiple dose study showed that in Chinese healthy subjects, the mean $C_{\max }$ of $80 \mathrm{mg}$ fluvastatin ER tablet once daily at single dose and multiple doses was 61.0 and $63.9 \mathrm{ng} / \mathrm{mL}$, which was $78.4 \%$ and $83.3 \%$ lower compared with that of $40 \mathrm{mg}$ fluvastatin IR capsule twice daily at single dose and multiple doses, respectively. The mean exposure with ER tablet (single dose and multiple dose $\mathrm{AUC}_{0-24 \mathrm{~h}}$ ) was 242 and $253 \mathrm{ng} \cdot \mathrm{h} / \mathrm{mL}$, which were $66.4 \%$ and $72.5 \%$ lower compared with IR capsule, respectively. However, the difference has no statistical significance at $P>0.05$ in reduction percent of cholesterol and triglyceride between group of $80 \mathrm{mg}$ fluvastatin ER tablet and group of $40 \mathrm{mg}$ fluvastatin IR capsule. The possible reason was that the time of effective concentration for $80 \mathrm{mg}$ fluvastatin ER was no shorter than that for $40 \mathrm{mg}$ fluvastatin IR.

The peak concentration was delayed for ER formulation of fluvastatin. In addition, there was less fluctuation from $C_{\max }$ to $C_{\min }$ for ER formulation of fluvastatin compared with IR formulation of fluvastatin.

\section{Conclusion}

The LC-tandem mass spectrometry method established for the determination of fluvastatin in human serum following administration of fluvastatin is simple, rapid, and showed to be highly reproducible and accurate. Compared with immediate-release formulation, $C_{\max }$ of fluvastatin extended-release tablet is reduced and $T_{\max }$ is prolonged, respectively. There is no accumulation for extended-release formulation after multiple doses. The difference has no statistical significance at $P>0.05$ in reduction percent of cholesterol and triglyceride between the two groups. Fluvastatin was well tolerated following single and multiple doses.

\section{References}

[1] E. Bruckert, M. P. Verpilleux, S. Dejager, and J. Isaacsohn, "Comparison of efficacy and safety assessment of Fluvastatin in patients $<65$ years versus $\geq 65$ years of age," American Journal of Cardiology, vol. 96, no. 8, pp. 1142-1148, 2005.

[2] V. L. Lanchote, A. Rocha, F. U. de Albuquerque, E. Barbosa Coelho, and P. S. Bonato, "Stereoselective analysis of fluvastatin in human plasma for pharmacokinetic studies," Journal of Chromatography B, vol. 765, no. 1, pp. 81-88, 2001.

[3] P. Ostadal, D. Alan, J. Vejvoda et al., "Immediate effect of fluvastatin on lipid levels in acute coronary syndrome," Molecular and Cellular Biochemistry, vol. 306, no. 1-2, pp. 1923, 2007.

[4] C. D. Scripture and J. A. Pieper, "Clinical pharmacokinetics of fluvastatin," Clinical Pharmacokinetics, vol. 40, no. 4, pp. 263281, 2001.

[5] J. L. Isaacsohn, J. LaSalle, G. Chao, and L. Gonasun, "Comparison of treatment with fluvastatin extended-release $80-\mathrm{mg}$ tablets and immediate-release 40-mg capsules in patients with primary hypercholesterolemia," Clinical Therapeutics, vol. 25, no. 3, pp. 904-918, 2003.

[6] D. Barilla, P. Prasad, M. Hubert, and K. Gumbhir-Shah, "Steady-state pharmacokinetics of fluvastatin in healthy subjects following a new extended release fluvastatin tablet, lescol XL," Biopharmaceutics and Drug Disposition, vol. 25, no. 2, pp. 51-59, 2004.

[7] C. M. Battantyne, F. Pazzucconi, X. Pintó et al., "Efficacy and tolerability of fluvastatin extended-release delivery system: a pooled analysis," Clinical Therapeutics, vol. 23, no. 2, pp. 177192, 2001. 

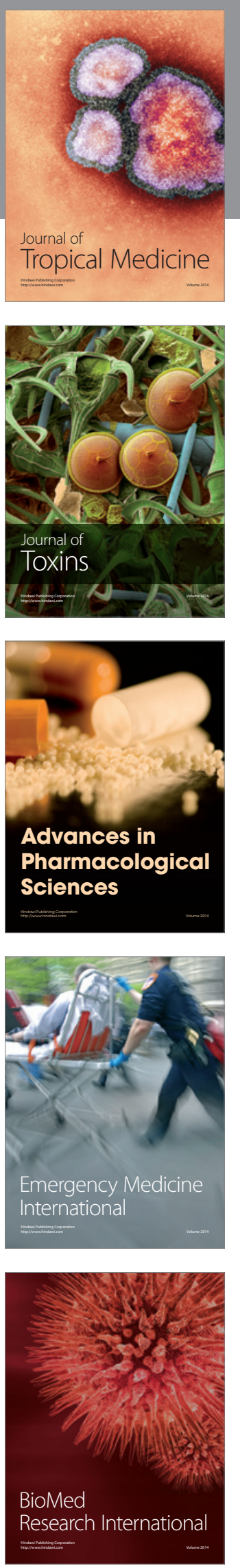
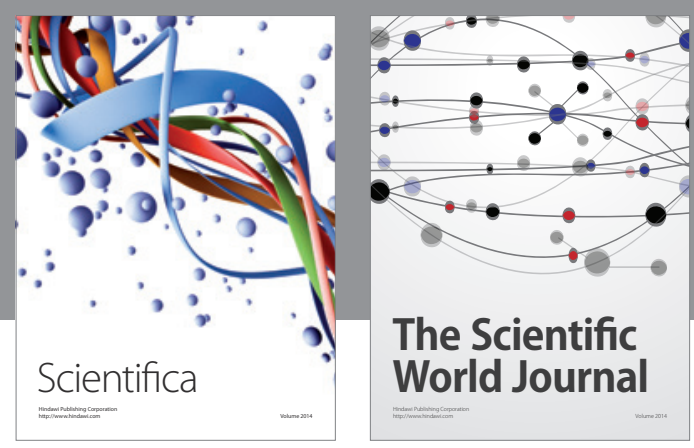

The Scientific World Journal
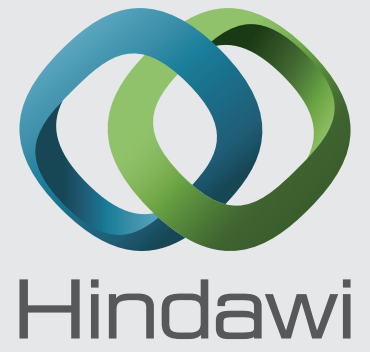

Submit your manuscripts at

http://www.hindawi.com
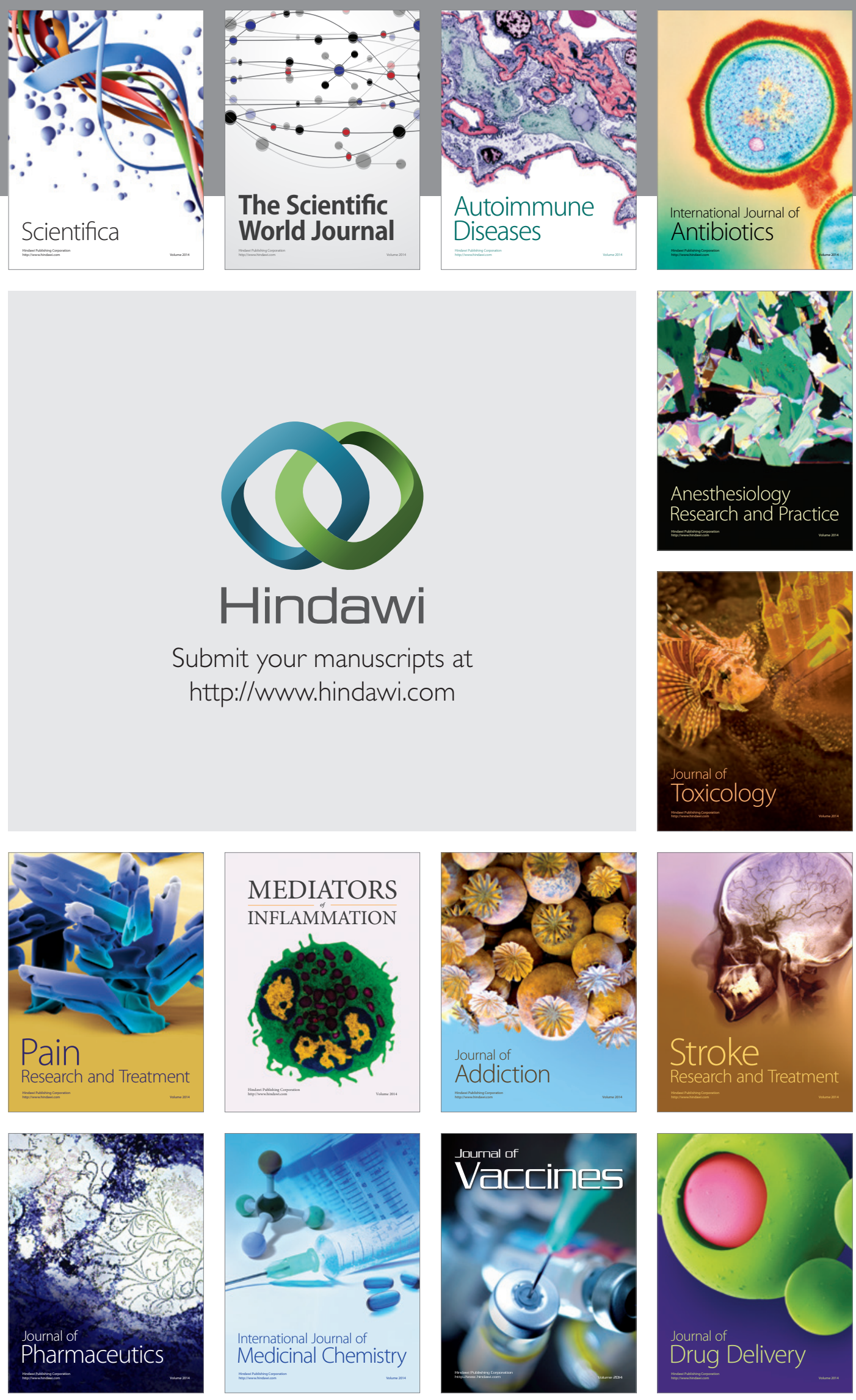